\title{
Mindfulness en estudiantes de medicina
}

\author{
Pere Oro, Montse Esquerda, Joan Viñas-Salas, Jorge Soler-González, Josep Pifarré
}

Resumen. Numerosos estudios muestran altos niveles de estrés y ansiedad en estudiantes de medicina y profesionales sanitarios, describiéndose múltiples factores relacionados (altos niveles de exigencia, contacto con la enfermedad y el sufrimiento), así como su resistencia a solicitar ayuda en caso de que la necesiten. Estos estresores se han asociado con agotamiento emocional, disminución de la empatía, consumo de tóxicos y riesgo de presentar problemas de salud mental. Se han definido diversos programas para mejorar el bienestar de los estudiantes. La presente revisión analiza la práctica del mindfulness (atención plena) como ayuda para disminuir el burnout y la fatiga por compasión, incrementar las habilidades de autocuidado, disminuir los niveles de ansiedad y mejorar el bienestar general en este colectivo. La práctica del mindfulness se ha relacionado asimismo con la mejora de las habilidades y competencias relacionadas con una buena práctica médica: ser consciente de los propios contenidos de consciencia, ponerse en posición de observador, capacidad empática, escucha activa, estar presente, metaconocimiento y metaconsciencia de lo que está ocurriendo en la interacción terapéutica y establecimiento de la alianza terapéutica (junto con su componente vincular). La revisión de la bibliografía apoya que se introduzca la práctica del mindfulness en el currículo formativo de los futuros médicos.

Palabras clave. Autocuidado. Educación médica. Estrés. Estudiantes de medicina. Mindfulness.

\section{Mindfulness in medical students}

Summary. Studies show high levels of stress and anxiety in medical students, describing multiple related factors. The stressors that affect them have been associated with emotional exhaustion, decreased empathy, toxic consumption and risk of mental health problems. This review shows how mindfulness practice can help to increase self-care skills and welfare in medical students, and their influence on stress, anxiety, burnout or compassion fatigue. Practicing mindfulness has also been associated with improved skills and competencies related to good medical practice: awareness, take an observer stance, empathic ability, active listening, being present and therapeutic alliance's establishment. The literature review supports the introduction of mindfulness practice in the training curriculum of future physicians.

Key words. Medical education. Medical students. Mindfulness. Self-care skills. Stress.

\section{Introducción}

Numerosos estudios [1-4] muestran altos niveles de estrés y ansiedad en estudiantes de medicina, describiéndose múltiples factores relacionados.

Por una parte, los estudiantes de medicina están sometidos a altos niveles de exigencia de rendimiento académico desde, al menos, el bachillerato, debido a la necesidad de obtener altas calificaciones para poder acceder a los estudios de medicina. Estas altas exigencias pueden implicar determinados tipos de comportamiento y de patrones relacionales (predisposición a situaciones de pobre relación social, reforzar patrones de autoexigencia y 'dureza' respecto a las necesidades habituales de una persona joven). Estas circunstancias pueden reforzar la manifestación de conductas obsesivas y otros patrones de comportamiento poco saludables [4].

Otro de los factores descritos, específico de los estudiantes de medicina, es el contacto, ya desde el inicio de las prácticas, con el dolor emocional, la enfermedad, el sufrimiento y la muerte. A medida que progresan en sus estudios van tomando contacto con las limitaciones y condicionantes propios de la práctica médica, las dificultades de comunicación y de competencias, la limitación de recursos o la mercantilización de la medicina. Se ha descrito una regresión del razonamiento moral y la empatía durante los estudios de medicina [5].

Los estudiantes de medicina y los profesionales de la salud en general están asimismo sometidos a un tipo de estrés relacionado con el cuidado de otros.
Facultad de Medicina; Universitat de Lleida (P. Oro, M. Esquerda, J. Viñas-Salas, J. Soler-González, J. Pifarré). Institut Borja de BioèticaURL (M. Esquerda, J. Viñas-Salas). IRB-Lleida (J. Viñas-Salas, J. SolerGonzález, J. Pifarré). Lleida, España.

Correspondencia:

Prof. Montse Esquerda. Facultad de Medicina. Universitat de Lleida. Rovira Roure, 44. E-25006 Lleida.

E-mail:

mesquerda@comll.cat

Conflicto de intereses: No declarado.

Competing interests: None declared.

(C) 2015 FEM 
Este estrés sostenido de una forma crónica puede dar lugar a lo que se ha denominado síndrome de burnout o del profesional 'quemado', asociado con las profesiones asistenciales y de relación con otros [6].

El burnout se caracteriza por una serie de síntomas como podrían ser agotamiento emocional, despersonalización (indiferencia e insensibilidad a ciertos aspectos del trabajo) y falta de realización personal. Se describen factores predisponentes de carácter personal, como poca experiencia, disponer de menos estrategias de afrontamiento al estrés, motivación muy elevada, exagerado sentido de la responsabilidad e idealización del trabajo.

Shapiro et al [7] recogen cómo el estrés puede incrementar el burnout en personal sanitario. Otros estudios explican que el burnout puede relacionarse con la falta de habilidades de cuidado personal de los médicos residentes y puede influir en que los pacientes reciban una atención deficiente [8].

Murray et al [9] efectuaron un seguimiento de médicos norteamericanos entre 1986 y 1997 y encontraron que iban declinando los aspectos relacionados con la satisfacción de su vida profesional. También existen estudios con estudiantes de medicina que relacionan el distrés asociado con el cumplimiento del currículo académico y el abuso de sustancias, la dificultad para relacionarse, el suicidio y el desgaste personal, lo que fomenta conductas de cinismo y otros inconvenientes [10]. Parece pues evidente que los estudiantes y profesionales de la salud tienen un riesgo de burnout y que sería adecuado entrenarlos en soportes que los ayuden con los numerosos estresores relacionados con su tarea.

Otro síndrome asociado con los estudiantes y profesionales de la salud es el de fatiga por compasión o desgaste por empatía [11]. La fatiga por compasión tendría una vertiente más personal que organizacional, sería una consecuencia de la preocupación por los otros o por su dolor emocional, podría aparecer repentinamente y sin previo aviso, y podría relacionarse con una especie de trauma secundario o vicario, debido a la relación con personas traumatizadas o que sufren.

Figley [11] comenta que podría ser algo parecido al coste a pagar por cuidar de otros: 'Los profesionales que escuchan de sus clientes historias de miedo, dolor y sufrimiento, pueden sentir un dolor, pena y sufrimiento similares, debido a que tienen a su cuidado a estas personas. Irónicamente, los terapeutas más efectivos son los más vulnerables a este efecto de reflejo o de contagio. Los que tienen gran capacidad para sentir y expresar empatía tienden a tener más riesgo de estrés por compasión. El estrés vicariante sería el que se generaría en estas situa- ciones y su mantenimiento prolongado estaría en la base de la fatiga por compasión, a la cual define como un 'estado de agotamiento y disfunción -biológica, psicológica y social- como resultado de prolongada exposición al estrés por compasión y a todo lo que éste evoca'.

A la luz de los conocimientos actuales, parecería que estos fenómenos podrían tener un nexo común, relacionado con la teoría de las neuronas espejo. En 1996, el equipo de Rizzolatti, estudiando el cerebro de monos, descubrió un grupo de neuronas que no sólo se activaban cuando el animal ejecutaba ciertos movimientos, sino que simplemente con contemplar a otros hacerlo, también entraban en acción. Las denominaron neuronas espejo o especulares.

El sistema de espejo permite hacer propias las acciones, sensaciones y emociones de los demás, te pone en el lugar del otro. La base de nuestro comportamiento social es que exista la capacidad de tener empatía e imaginar lo que el otro está pensando.

Rizzolatti et al [12] afirman que este tipo de neuronas permiten captar las intenciones de los otros. Estas neuronas se activan incluso aunque no veamos la acción, cuando hay una representación mental de ella. Su puesta en marcha corresponde con las ideas. Las neuronas espejo permiten entender la mente de los demás no sólo a través de un razonamiento conceptual, sino mediante la simulación directa, sintiendo, no pensando.

Asimismo, actualmente un alto número de estudiantes son mujeres, con lo que podrían sumarse problemas de adaptación adicionales [13,14] por dificultades en los modelos de enculturación del rol profesional.

Otro factor de riesgo adicional puede ser la resistencia a admitir, si se da el caso, de que pueden necesitar ayuda: serán médicos, no pueden permitirse padecer ciertas dolencias, les resulta muy difícil aceptarlo y pueden resistirse a solicitar dicha ayuda, por miedo a que pueda perjudicarlos profesionalmente.

En consecuencia, se ha descrito un incremento del agotamiento emocional, disminución de empatía, riesgo de consumo de tóxicos y aumento del riesgo de presentar problemas de salud mental. El estudio de Salamero et al [15] presenta una buena descripción en una muestra de estudiantes de medicina de nuestro contexto. En su estudio se detectan niveles importantes de distrés emocional y riego elevado de sufrir trastornos psicopatológicos, especialmente en las mujeres.

Se han intentado diversos programas estructurados para mejorar el bienestar de los estudiantes de medicina y el desarrollo de estrategias preventi- 
vas para cuando sean profesionales de la salud. Entre ellos nos centraremos en los programas basados en mindfulness. En este artículo se efectúa una revisión de dichos programas en relación con los estudiantes de medicina, incluyendo los publicados hasta el primer trimestre de 2015.

\section{¿Qué es mindfulness?}

La traducción más extendida de la palabra 'mindfulness' en nuestro idioma es atención plena o consciencia plena. Mindfulness es la traducción inglesa del término 'sati', que en el idioma pali (antigua lengua índica) significaría consciencia, atención y recuerdo.

Para Kabat-Zinn [16], pionero y creador del programa Mindfulness Based Stress Reduction (MBSR), mindfulness significa prestar atención de un modo particular: centrado en el momento presente, a propósito, y sin establecer juicios de valor. Incluye una cualidad amable y compasiva en la persona que presta atención.

Existen diferencias a la hora de definir mindfulness como un constructo psicológico: para unos autores es unidimensional; para otros, bidimensional, y para otros, multidimensional. Estas diferencias han comportado la existencia de diferentes instrumentos para medirlo, algunos de los cuales se han adaptados al castellano y validado en nuestro contexto [17-19]. Los estudios, incluso en nuestro contexto [20], parecen apoyar que los enfoques no unidimensionales recogen mejor la complejidad e integridad del constructo.

Esta práctica se desarrolla mediante una serie de intervenciones estructuradas, formales e informales. Los programas más conocidos incluyen una combinación de ejercicios (exploración corporal, estiramientos, meditaciones guiadas, visualizaciones...) para desarrollar una mayor consciencia del cuerpo, las emociones, los pensamientos y el sentido del 'yo'.

Kristeller [21] describe un modelo de seis dominios en los que tendría influencia la práctica del mindfulness; éstos serían el cognitivo, el fisiológico, el emocional, el conductual, la relación con sí mismo y con los demás, y el espiritual.

De manera más específica, la práctica de mindfulness se ha relacionado con la neuroplasticidad, la integración neural y el equilibrio neurológico [2225]. Posibilita el responder de manera más espontánea, más natural, con discernimiento, en lugar de actuar de forma muy restringida y determinada por los condicionantes internos y externos que nos afectan. Se ha relacionado la práctica del mindfulness con incrementos del bienestar general, mejoras en el funcionamiento corporal, salud, respuesta inmunológica, bienestar físico, claridad mental y reducción de tensión corporal, por citar algunos. De forma muy resumida, dos de las más importantes funciones atribuidas al mindfulness son facilitar y favorecer un equilibro cognitivo y afectivo.

Treadway y Lazar [26] recogen una serie de resultados relacionados con la práctica de la meditación mindfulness: más tiempo viviendo el momento presente con mayor capacidad para tomar distancia de las emociones negativas, mayor afecto positivo como un patrón emocionalmente estable de actividad cerebral, menos reactividad al estrés y mayor vitalidad cognitiva; incluso existen estudios que han encontrado evidencia de que la práctica del mindfulness puede tener efectos neuroprotectores y reducir el deterioro cognitivo asociado con el envejecimiento [27].

Se han descrito numerosas aplicaciones del mindfulness en diferentes usos terapéuticos, desde los programas grupales y más genéricos de reducción de estrés, a desarrollos enfocados a otros trastornos específicos y que pueden comportar intervenciones grupales o individuales. Está incorporado a terapias de diferentes modelos teóricos (cognitivo-conductual, psicodinámico, humanista...). A modo de ejemplo puede consultarse el manual de Miró y Simón [28].

\section{Mindfulness en estudiantes y profesionales de medicina}

La práctica del mindfulness se ha demostrado útil también para los propios profesionales de ciencias de la salud y, más en concreto, los médicos y estudiantes de medicina [29-57]. Tres son los aspectos importantes: uso del mindfulness para mejorar habilidades de cuidado personal, uso para mejorar habilidades y competencias propias de su trabajo, y la aplicación explícita de técnicas o terapias basadas en mindfulness con los pacientes. Este último punto no se desarrollará en esta revisión.

\section{Uso del mindfulness para mejorar habilidades de cuidado personal}

Como se ha comentado, los estudiantes y profesionales de medicina acostumbran a estar sometidos a un alto nivel de exigencia y deben tratar con un tipo de problemáticas que los puede hacer vulnerables a padecer situaciones que afecten a la correcta gestión de sus emociones y a su propia salud entendida en un sentido integral, tanto psíquico como somático. 
En uno de los programas pioneros, Shapiro et al [29] aplicaron el programa MBSR de Kabat-Zinn [16] a una muestra de estudiantes de medicina y encontraron que disminuían sus niveles de ansiedad y otros trastornos del estado de ánimo, se incrementaban los niveles de empatía y las puntuaciones que mediaban aspectos relacionados con la espiritualidad.

Numerosos estudios posteriores han mostrado resultados prometedores. Rosenzweig et al [30] aplicaron el programa MBSR a una muestra de estudiantes de medicina y hallaron disminuciones significativas de su nivel de alteración emocional.

Hassed et al [31] han aplicado un programa de mejora de la salud, basado en mindfulness, en estudiantes de medicina, han estudiado su influencia sobre sus niveles de ansiedad, bienestar y otras variables, $y$ han comprobado sus efectos en periodos especialmente estresantes como son los de exámenes; han obtenido resultados positivos significativos en su estudio.

De Vibe et al [32] han investigado los efectos de un programa de mindfulness de siete semanas de duración en estudiantes de medicina y psicología; han encontrado mejoras en los estudiantes de medicina en agotamiento mental, estrés relacionado con los estudios, bienestar subjetivo y mindfulness. Lo particular de su estudio es que la mejoría es más significativa en estudiantes de sexo femenino: los autores sugieren que estas diferencias pueden deberse a procesos biológicos relacionados con la forma en que se experimentan las emociones, además de procesos de socialización asociados a cómo las emociones y el estrés se expresan en función del sexo.

Kuhlmann et al [33] han desarrollado un protocolo de investigación muy riguroso para comprobar la efectividad de un programa que combina mindfulness y terapia cognitiva en estudiantes de medicina. Utilizan tres grupos con asignación aleatoria: el de mindfulness y terapia cognitiva, uno de entrenamiento autógeno y otro de control; el periodo de evaluación se prolonga tras la finalización del programa, estando previstas mediciones transcurridos uno y cinco años. Todavía no se dispone de resultados.

Greeson et al [34] han adaptado un taller de cuatro semanas de duración basado en el trabajo de habilidades mente-cuerpo para estudiantes de medicina. Lo han aplicado en estudiantes voluntarios de forma extracurricular y han hallado buena aceptación y mejora en habilidades de autocuidado, incremento de mindfulness y disminución de niveles de estrés.

Phang et al [35] han aplicado un programa de reducción de estrés basado en mindfulness de cinco semanas de duración a estudiantes de medicina. Una semana después de la intervención existían diferencias significativas, con respecto al grupo control, en medidas de mindfulness, estrés percibido, distrés psíquico y autoeficacia. Repitieron las mediciones seis meses después de la intervención y la única diferencia que se mantenía con respecto al grupo de control era la mejora en autoeficacia.

Intentando alcanzar una mayor economía de medios, Kemper y Yun [36] han utilizado un método híbrido (un curso de MBSR disponible online con el apoyo de un médico residente practicante, pero sin instrucción formal) para entrenar a un pequeño grupo de estudiantes de medicina. En la misma línea, Warnecke et al [37] han aplicado un programa de práctica de mindfulness para entrenar a estudiantes avanzados de medicina con objeto de disminuir su nivel de estrés, obteniendo resultados positivos. La particularidad de su método reside en que solamente facilitaban un CD de audio a los estudiantes, con el que debían practicar en casa diariamente durante ocho semanas.

Van Dijk et al [38] aportan un dato interesante en su estudio sobre aplicación de entrenamiento en mindfulness con estudiantes de medicina. Encuentran que los estudiantes interesados en participar en los entrenamientos de mindfulness y los que realmente participan muestran puntuaciones superiores en distrés psicológico y neuroticismo en comparación con los que no muestran interés en dichos programas. Estos resultados refuerzan la idea de la importancia de la aleatoriedad en la asignación al grupo experimental o control en estos estudios y que, cuando se ofrecen estos programas en las facultades de medicina, parece que despiertan mayor interés en los estudiantes que mayor beneficio pueden obtener de ellos.

Alguno de estos programas se ha aplicado también en médicos de atención primaria. Krasner et al [39] han desarrollado un programa basado en mindfulness, comunicación y autoconocimiento. Incluye material didáctico, meditación mindfulness formal (escáner corporal, meditación sentada, paseo meditativo y movimientos conscientes), prácticas narrativas con ejercicios de indagación apreciativa y deliberaciones en grupo. Los participantes consiguieron mejoras en su bienestar y en las actitudes asociadas con una atención centrada en el paciente.

Irving et al [40] efectuaron una revisión de estudios empíricos en los que se utilizaba MBSR para incrementar el bienestar y reducir el estrés en profesionales de la salud (se incluían enfermeros, médicos, psicólogos...). Afirman que existe evidencia empírica de los beneficios de este programa en la salud física y mental de los clínicos, aunque la ma- 
yoría de estudios utilizan medidas de autoinforme y muy pocos incluyen medidas de registros psicofisiológicos, como cortisol en saliva, u hormonales.

En nuestro entorno, Martín et al [41] han evaluado la efectividad, en un periodo dilatado (un año), de un programa de MBSR dirigido a profesionales de atención primaria. Han encontrado mejoras significativas en burnout, alteración emocional y otras variables, destacando que se fomentan las actitudes dirigidas al autocuidado.

El programa MBSR de Kabat-Zinn se ha aplicado también a una muestra de estudiantes de psicoterapia [42] y se ha hallado que estas prácticas, que los autores denominan 'de autocuidado', consiguieron reducciones significativas en estrés, afecto negativo, rumiación y ansiedad (estado y rasgo). Así mismo, obtuvieron incrementos en afecto positivo y autocompasión. Encontraron incremento en la medida del constructo mindfulness y comprobaron si podría ser una variable predictiva de las mejoras en las otras variables medidas.

En el trabajo ya comentado de Shapiro et al [7] se expone que el programa MBSR ofrece un modelo concreto, breve y equilibrado en el sentido de coste-resultados para poder aplicarse fácilmente en clínicas y hospitales, a la vez que destacan la necesidad de adoptar medidas y proporciona recursos para aliviar el estrés en estos colectivos.

Con respecto a la importancia de la práctica regular de los ejercicios en casa, estos programas acostumbran a tener un alto nivel de exigencia en este aspecto; por ejemplo, el programa MBSR de KabatZinn demanda unos 45 minutos de práctica diaria en casa en determinadas condiciones de silencio, recogimiento..., que pueden resultar difíciles de conseguir en los hogares actuales.

Vettese et al [43] efectuaron una revisión de 98 estudios, de los cuales 24 evaluaron la asociación entre práctica de ejercicios en casa y los resultados de mejora obtenidos; en 13 trabajos (54\%) se encontró al menos apoyo parcial a los beneficios de la práctica fuera de las sesiones presenciales. Llama la atención la diferencia entre la importancia que se concede a dicha práctica en la metodología de estos programas y la cuestionable evidencia acerca de su utilidad en la mejora de las sintomatologías, por lo que es importante validar la utilidad real de estas prácticas para poder dimensionarlas adecuadamente.

Dobkin y Hutchinson [44] efectúan una revisión acerca de las facultades de medicina que incorporan formación en mindfulness a sus estudiantes y residentes. Encuentran que 14 de ellas ya incluyen esta formación con diferentes formatos y metodologías, y dos han integrado mindfulness en sus pla- nes de estudios. Destacan la utilidad de estos programas para reducir el burnout y la fatiga por compasión, disminuir el estrés y aumentar el bienestar psicológico. También destacan que estos médicos disponen de mejores recursos para fomentar el bienestar de sus pacientes.

\section{Uso del mindfulness para mejorar \\ habilidades y competencias relacionadas con una buena práctica médica}

Entre las habilidades que debe poseer un buen profesional de la salud podemos citar la capacidad empática, la escucha activa y el estar presente, demandando todo ello el metaconocimiento y metaconsciencia de lo que está ocurriendo en la interacción terapéutica y el establecimiento de una buena alianza terapéutica (junto con su componente vincular). Diversos estudios muestran que la práctica del mindfulness puede mejorar estas habilidades y las competencias de los futuros médicos.

Los estudios en este ámbito se han realizado principalmente con estudiantes y profesionales de psicología, aunque también los hay en el ámbito de los estudiantes y profesionales de la medicina.

Cohen y Miller [45] han desarrollado un programa de seis semanas, siguiendo el modelo de MBSR, para entrenar estudiantes de psicología en mindfulness, enfatizando el aspecto de consciencia de la relación. Los resultados obtenidos sugieren un efecto positivo en mindfulness, estrés percibido, conexión social, inteligencia emocional y ansiedad. Conceden importancia a los cambios en bienestar interpersonal, pues lo consideran un beneficio esencial para futuros profesionales de la salud mental y lo consideran un complemento útil para la formación clínica.

Outram y Kelly [46] resaltan la importancia que tiene para los médicos el aprender a enfrentarse al sufrimiento debido a la enfermedad y a la vida, a la culpa y autoexigencia excesivas, y al deseo de perfeccionismo que impide compartir los problemas y que aísla de los demás. Resulta notable la coincidencia con los componentes de la autocompasión que Neff [47] ha descrito: amabilidad con uno mismo sin autocrítica, sentirse parte de la humanidad y no aislado, y mindfulness o apertura de conciencia sin ensimismamiento. Desde otro modelo teórico, Gilbert [48] describe el subsistema neuronal de calma y satisfacción, relacionado con la oxitocina y las endorfinas. Tanto el modelo de Neff y Germer (difundido en nuestro país por Vicente Simón y Marta Alonso) como el de Gilbert inciden en la importancia de desarrollar la compasión (brindar cuidado, 
consuelo y serenidad al que sufre, fracasa o se siente inadecuado) y la autocompasión (darnos el mismo cuidado, consuelo y serenidad que daríamos a otro cuando sufrimos, fracasamos o nos sentimos inadecuados). La importancia de la compasión y la autocompasión hace que su práctica, cada vez más, forme parte de manera explícita de los programas de entrenamiento de mindfulness más actuales, pudiéndose hablar con propiedad de programas de mindfulness y compasión.

Wexler [49] ha estudiado la relación entre las habilidades mindfulness del terapeuta y la calidad de la alianza terapéutica, medida en el terapeuta y el paciente, y ha encontrado una correlación positiva significativa entre estas dos variables.

Grepmair et al [50] estudiaron si entrenar en mindfulness a psicoterapeutas en formación influía en los resultados que obtenían en las psicoterapias que realizaban a sus pacientes. Los pacientes de los psicoterapeutas que practicaban meditación obtuvieron mejores puntuaciones, con diferencias significativas, en clarificación y resolución de problemas; también se redujeron sus síntomas de manera significativa en somatización, evitación social, obsesividad, ansiedad, ira, ansiedad fóbica, pensamiento paranoide y psicoticismo.

Hick [51] enfatiza la importancia del mindfulness en el contexto psicoterapéutico, considerándolo muy importante para el terapeuta. Destaca su utilidad para que el terapeuta pueda moverse desde el 'modo hacer' al 'modo ser'; afirma que este 'modo ser' ayuda a dirigir al terapeuta hacia un encuentro directo con el paciente, en el 'aquí y ahora. Plantea que la relación terapéutica es un factor muy importante en el éxito de una terapia y que mindfulness contribuye al desarrollo de los diferentes componentes de una buena relación terapéutica, como son la empatía, la escucha atenta y la compasión.

Linehan [52] postula que mindfulness, más que una intervención, debería considerarse una estrategia psicoterapéutica, pues las actitudes y conductas del terapeuta son más importantes para la relación terapéutica que las habilidades que intenta enseñar al paciente.

Robins [53] resalta la importancia para los terapeutas de practicar mindfulness, en un contexto de terapia dialéctico-conductual para pacientes con trastorno límite de la personalidad, y plantea que tiene los siguientes efectos:

- Incrementar la habilidad para observar y describir la conducta de los pacientes en las sesiones de una manera no crítica (sin juzgar).

- Incrementar la habilidad para permanecer focalizados en la tarea y en el momento presente.
- Ayudar a regular sus propias emociones durante las sesiones, manteniéndose consciente en su propia respiración.

- Manejar sus propias opiniones acerca de su competencia como profesional (está obrando como mejor sabe en cada momento, poder rectificar en beneficio del paciente...).

- Ayudar a desarrollar una actitud de desapego, esforzándose por ayudar al paciente a alcanzar ciertas metas y, al mismo tiempo, no permanecer apegado a estos resultados, disminuyendo así su grado de sufrimiento si no pueden conseguirse.

Warde et al [54] han utilizado un entrenamiento para desarrollar habilidades muy necesarias en la práctica médica, especialmente en entornos de poblaciones vulnerables. Entre estas habilidades están el liderazgo, dar apoyo y soporte, el trabajo en equipo, mindfulness y el autocuidado. En esta línea, Epstein y Krasner [55] también destacan la importancia del entrenamiento en mindfulness para fomentar la resiliencia (entendida como capacidad de responder al estrés de una manera saludable, de tal manera que se logren los objetivos con un coste psicológico y físico mínimo) en los médicos, pues permite mejorar la calidad de la atención, del cuidado y su sostenibilidad. Esta resiliencia dependería de factores individuales, de grupo e institucionales; entre los factores individuales estarían la capacidad de mindfulness, el autochequeo, el establecimiento de límites y las actitudes que promueven la participación constructiva y saludable con -en lugar de retirarse de- los a menudo difíciles retos en el trabajo.

Siegel [56], refiriéndose a la plasticidad neuronal que provoca la práctica de mindfulness, afirma que los cambios que se producen en el cerebro cuando estamos sintonizados emocionalmente con nuestros estados internos durante la meditación parecen correlacionarse con las zonas del cerebro activas cuando nos sentimos conectados con los demás (sintonía interpersonal e intrapersonal), y esto sugiere que los terapeutas pueden entrenar su cerebro para que sea más efectivo desde el punto de vista terapéutico, gracias a la meditación mindfulness.

Dobkin y Laliberté [57] inciden en un aspecto no tenido en cuenta hasta ahora: cómo las habilidades mindfulness pueden ayudar a ser un buen docente, un buen profesor, para los estudiantes de medicina. Los autores proponen ejemplos de conductas mindfulness en residentes de psiquiatría que forman a estudiantes y afirman que están en línea con la filosofía humanista y el 'modo ser' defendidos por Karl Jaspers. 
Todos estos aspectos son muy importantes para la práctica clínica en los profesionales médicos, puesto que la atención receptiva que caracteriza el mindfulness puede potenciar una mayor capacidad o voluntad de interesarse por los pensamientos y las emociones de la otra persona que participa en la comunicación, y también puede mejorar la capacidad de prestar atención al comportamiento no verbal del otro. Al mismo tiempo, esa persona puede ser más consciente de sus propias respuestas cognitivas, emocionales y verbales en la comunicación.

\section{Conclusiones}

Vistas las aportaciones que puede realizar el mindfulness en el campo de la medicina, deberían seguirse con interés las experiencias en las que se está introduciendo la enseñanza y la práctica del mindfulness en el currículo formativo de las diferentes escuelas y facultades de medicina del mundo, identificar las mejores prácticas en función de sus resultados e intentar introducirlas en las facultades de medicina.

Un aspecto importante es determinar el tiempo de práctica personal necesario para obtener resultados, dada las discrepancias existentes al respecto, así como la efectividad de los diferentes componentes (practica informal, estiramientos físicos, exploración corporal, meditaciones guiadas, visualizaciones, meditaciones de 'campo abierto'...) que se utilizan en los programas de mindfulness. Puede ser de interés identificar las aportaciones que puede realizar la práctica del mindfulness a las competencias profesionales básicas detectadas por grupos de expertos en los diferentes estudios al respecto [58, 59], así como la utilidad del mindfulness en favorecer una buena transición desde las escuelas y facultades a la vida profesional [60].

\section{Bibliografía}

1. Abdulghani HM, Irshad M, Al Zunitan MA, Al Sulihem AA, Al Dehaim MA, Al Esefir WA, et al. Prevalence of stress in junior doctors during their internship training: a cross-sectional study of three Saudi medical colleges' hospitals. Neuropsychiatr Dis Treat 2014; 10: 1879-86.

2. Erogul M, Singer G, McIntyre T, Stefanov DG. Abridged mindfulness intervention to support wellness in first-year medical students. Teach Learn Med 2014; 26: 350-6.

3. Wasserman JA, Fitzgerald JE, Sunny MA, Cole M, Suminski RR, Dougherty JJ. Nonmedical use of stimulants among medical students. J Am Osteopath Assoc 2014; 114: 643-53.

4. Sender-Romeo R, Salamero-Baró M. Programa de atención psicológica para los alumnos de la Facultad de Medicina de la Universidad de Barcelona. Educ Med 2007; 10 :58-63.

5. Hren D, Marusic M, Marusic A. Regression of moral reasoning during medical education: Combined design study to evaluate the effect of clinical study years. PLoS One 2011; 6: e17406.
6. Maslach C. Burnout: the cost of caring. Englewood Cliffs, NJ: Prentice-Hall; 1982.

7. Shapiro SL, Astin JA, Bishop SR, Cordova M. MindfulnessBased Stress Reduction for health care professionals: results from a randomized trial. Int J Stress Manag 2005; 12: 164-76.

8. Shanafelt TD, Bradley KA, Wipf JE, Back AL. Burnout and self-reported patient care in an internal medicine residency program. Ann Intern Med 2002; 136: 358-67.

9. Murray A, Montgomery JE, Chang H, Rogers WH, Inui T, Safran DG. Doctor discontent. A comparison of physician satisfaction in different delivery system settings, 1986 and 1997. J Gen Intern Med 2001; 16: 452-9.

10. Dyrbye LN, Thomas MR, Shanafelt TD. Systematic review of depression, anxiety, and other indicators of psychological distress among U.S. and Canadian medical students. Acad Med 2006; 81: 354-73.

11. Figley CR, ed. Compassion fatigue: coping with secondary traumatic stress disorder in those who treat the traumatized. London: Brunner-Routledge; 1995.

12. Rizzolatti G, Fogassi L, Gallese V. The mirror neuron system: a motor-based mechanism for action and intention understanding. In Gazzaniga MS, ed. The cognitive neuroscience. 3 ed. Cambridge, MA: MIT Press; 2009. p. 625-40.

13. Smulyan L. Redefining self and success: becoming teachers and doctors. Gender \& Education 2004; 16: 225-45.

14. Blanch DC, Hall JA, Roter DL, Frankel RM. Medical student gender and issues of confidence. Patient Educ Couns 2008; 72: $374-81$

15. Salamero M, Baranda L, Mitjans A, Baillés E, Cámara M, Parramon G, et al. Estudi sobre la salut, estils de vida $i$ condicionants acadèmics dels estudiants de medicina de Catalunya. Barcelona: Fundació Galatea; 2012.

16. Kabat-Zinn J. Full catastrophe living: using the wisdom of your body and mind to face stress, pain and illness. New York: Dell; 1990.

17. Soler J, Tejedor R, Feliu-Sol A, Pascual JC, Cebolla A, Soriano J, et al. Propiedades psicométricas de la versión española de la escala Mindful Attention Awareness Scale (MAAS). Actas Esp Psiquiatr 2012; 40: 18-25.

18. Tejedor R, Feliu-Soler A, Pascual JC, Cebolla A, Portella MJ Trujols J, et al. Propiedades psicométricas de la versión española de la Philadelphia Mindfulness Scale. Rev Psiquiatr Salud Ment 2014; 7: 157-65.

19. Cebolla A, García-Palacios A, Soler J, Guillén V, Baños R, Botella C. Psychometric properties of the Spanish validation of the Five Facets of Mindfulness Questionnaire (FFMQ). Eur J Psychiatry 2012; 26: 118-26.

20. Inchausti F, Prieto G, Delgado AR Análisis Rasch de la versión española de la escala Mindful Attention Awareness Scale en una muestra clínica. Rev Psiquiatr Salud Ment 2014; 7: 32-41.

21. Kristeller JL Mindfulness meditation. In Lehrer PM, Woolfolk RL, Sime WE, eds. Principles and practice of stress management. 3 ed. New York: Guilford Press; 2007.

22. Tang YY, Hölzel BK, Posner MI. The neuroscience of mindfulness meditation. Nat Rev Neurosci 2015; 16: 213-25.

23. Ricard M, Lutz A, Davidson RJ. En el cerebro del meditador. Investigación y Ciencia 2015; 18-25.

24. Vago DR, Silbersweig DA. Self-awareness, self-regulation, and self-transcendence (S-ART): a framework for understanding the neurobiological mechanisms of mindfulness. Front Hum Neurosci 2012; 6: 296.

25. Malinowski P. Neural mechanisms of attentional control in mindfulness meditation. Front Neurosci 2013; 7:8.

26. Treadway MT, Lazar SW. The neurobiology of mindfulness. In Didonna F, ed. Clinical handbook of mindfulness. New York: Springer; 2009. p. 45-57.

27. Jacobs T, Epel E, Lin J, Balcjburn EH, Wolkowitz OM, Bridwell DA, et al. Intensive meditation training, immune cell telomerase activity, and psychological mediators. Psychoneuroendocrinology 2011; 36: 664-81.

28. Miró MT, Simón V, eds. Mindfulness en la práctica clínica. Bilbao: Desclée de Brouwer; 2012.

29. Shapiro SL, Schwartz GE, Bonner G. Effects of mindfulness- 
based stress reduction on medical and premedical students. J Behav Med 1998; 21: 581-99.

30. Rosenzweig S, Reibel DK, Greeson JM, Brainard GC, Hojat M. Mindfulness-based stress reduction lowers psychological distress in medical students. Teach Learn Med 2003; 15: 88-92.

31. Hassed C, De Lisle S, Sullivan G, Pier C. Enhancing the health of medical students: outcomes of an integrated mindfulness and lifestyle program. Adv Health Sci Educ Theory Pract 2009, 14: 387-98.

32. De Vibe M, Solhaug I, Tyssen R, Friborg O, Rosenvinge JH, Sørlie T, et al. Mindfulness training for stress management: a randomised controlled study of medical and psychology students. BMC Med Educ 2013; 13: 107.

33. Kuhlmann SM, Bürger A, Esser G, Hammerle F. A mindfulnessbased stress prevention training for medical students (MediMind): study protocol for a randomized controlled trial. Trials 2015; 16: 40.

34. Greeson JM, Toohey MJ, Pearce MJ. An adapted, four-week mind-body skills group for medical students: reducing stress, increasing mindfulness, and enhancing self-care. Explore (NY) 2015; 11: 186-92.

35. Phang CK, Mukhtar F, Ibrahim N, Keng SL, Mohd Sidik S. Effects of a brief mindfulness-based intervention program for stress management among medical students: the Mindful-Gym randomized controlled study. Adv Health Sci Educ Theory Pract 2015; Feb 20. [Epub ahead of print].

36. Kemper KJ, Yun J. Group online mindfulness training: proof of concept. J Evid Based Complementary Altern Med 2015; 20: 73-5.

37. Warnecke E, Quinn S, Ogden K, Towle N, Nelson MR. A randomised controlled trial of the effects of mindfulness practice on medical student stress levels. Med Educ 2011; 45: 381-8.

38. Van Dijk I, Lucassen PL, Speckens AE. Mindfulness training for medical students in their clinical clerkships: two crosssectional studies exploring interest and participation. BMC Med Educ 2015; 15: 24.

39. Krasner MS, Epstein RM, Beckman H, Suchman AL, Chapman B, Mooney CJ, et al. Association of an educational program in mindful communication with burnout, empathy, and attitudes among primary care physicians. JAMA 2009; 302: 1284-93.

40. Irving JA, Dobkin PL, Park J. Cultivating mindfulness in health care professionals: a review of empirical studies of mindfulness-based stress reduction (MBSR). Complement Ther Clin Pract 2009; 15: 61-6.

41. Martín A, Rodríguez T, Pujol-Ribera E, Berenguera A, Moix J. Evaluación de la efectividad de un programa de mindfulness en profesionales de atención primaria. Gac Sanit 2013; 27: 521-8.

42. Shapiro SL, Brown KW, Biegel GM. Teaching self-care to caregivers: effects of mindfulness-based stress reduction on the mental health of therapists in training. Train Educ Prof Psychol 2007; 1: 105-15.

43. Vettese LC, Toneatto T, Stea JN, Nguyen L, Wang JJ. Do mindfulness meditation participants do their homework?
And does it make a difference? A review of the empirical evidence. J Cogn Psychother 2009; 23: 198-225.

44. Dobkin PL, Hutchinson TA. Teaching mindfulness in medical school: where are we now and where are we going? Med Educ 2013; 47: 768-79.

45. Cohen JS, Miller LJ. Interpersonal mindfulness training for well-being: a pilot study with psychology graduate students. Teach Coll Rec 2009; 111: 2760-74.

46. Outram S, Kelly B 'You teach us to listen,... but you don't teach us about suffering': self-care and resilience strategies in medical school curricula. Perspect Med Educ 2014; 3: 371-8.

47. Neff KD, Germer CK. A pilot study and randomized controlled trial of the mindful self-compassion program. J Clin Psychol 2013; 69: 28-44.

48. Gilbert P. Evolved minds and compassion in the therapeutic relationship. In Gilbert P, Leahy RL, eds. The therapeutic relationship in the cognitive behavioral psychotherapies. London: Routledge; 2009. p. 106-42.

49. Wexler J. The relationship between therapist mindfulness and the therapeutic alliance. Diss Abstr Int Sect B Sci Eng 2006; 67: 2848.

50. Grepmair L, Mitterlehner F, Loew T, Bachler E, Rother W, Nickel M. Promoting mindfulness in psychotherapists in training influences the treatment results of their patients: a randomized, double-blind, controlled study. Psychother Psychosom 2007; 76: 332-8.

51. Hick SF. Cultivating the therapeutic relationship. The role of mindfulness. In Hick SF, Bien T, eds. Mindfulness and the therapeutic relationship. New York: Guilford Press; 2008.

52. Linehan MM. Cognitive-behavioral treatment of borderline personality disorder. New York: Guilford Press; 1993.

53. Robins CJ. Zen principles and mindfulness practice in dialectical behavior therapy. Cogn Behav Pract 2002; 9: 50-7.

54. Warde CM, Vermillion M, Uijtdehaage S. A medical student leadership course led to teamwork, advocacy, and mindfulness. Fam Med 2014; 46: 459-62.

55. Epstein RM, Krasner MS. Physician resilience: what it means, why it matters, and how to promote it. Acad Med 2013; 88: 301-3.

56. Siegel DJ. The Mindful brain: reflection and attunement in the cultivation of well-being. New York: Norton \& Co.; 2007.

57. Dobkin PL, Laliberté V. Being a mindful clinical teacher: can mindfulness enhance education in a clinical setting? Med Teach 2014; 36: 347-52.

58. Prat J, Carreras J, Branda L, Miralles R, Fenoll MR, Rodríguez $\mathrm{S}$, et al. Competències professionals bàsiques comunes dels llicenciats en medicina formats a les universitats de Catalunya. Barcelona: AQUA; 2004.

59. Core Committee. Institute for International Medical Education Global minimum essential requirements in medical education. Med Teach 2002; 24: 130-5.

60. Bullock A, Fox F, Barnes R, Doran N, Hardyman W, Moss D, et al. Transitions in medicine: trainee doctor stress and support mechanisms. J Work Learn 2013; 25: 368-82. 